\title{
TIME SYNCHRONIZATION ALGORITHMS FOR IEEE 802.11 OFDM SYSTEMS
}

\author{
Pei Xiao*, Colin Cowan*, T. Ratnarajah*, Anthony Fagan ${ }^{\dagger}$ \\ *The Institute of Electronics, Communications and Information Technology \\ Queen's University Belfast, BT3 9DT, United Kingdom \\ E-mail: \{pei.xiao,c.cowan, t.ratnarajah\}@ecit.qub.ac.uk \\ ${ }^{\dagger}$ Dept. of Electronic and Electrical Engineering, University College Dublin, Dublin 4, Ireland \\ E-mail: tony. fagan@ucd.ie
}

Keywords: IEEE 802.11, OFDM, Sychronization

\begin{abstract}
Time synchronization algorithms for OFDM systems using the short and long training symbols are investigated in this paper. We only consider efficient low complexity schemes that are feasible for practical implementations. Different algorithms are compared in the context of IEEE 802.11 Wireless Local Area Network (WLAN) systems. Based on the simulation results, some recommendations are made as to how the short and long training symbols can be effectively utilized for synchronization purposes.
\end{abstract}

\section{Introduction}

Orthogonal frequency division multiplexing (OFDM) has been widely adopted in broadband wireless communication systems, such as IEEE 802.11a [1], due to its robustness against the effects of multi-path propagation. However, OFDM systems are more sensitive to synchronization errors than single carrier systems. Timing and frequency synchronizations are crucial parts of OFDM receiver design, and often give rise to challenging problems $[2,3,4]$.

Synchronization of an OFDM signal requires finding the symbol timing and carrier frequency offset. Incorrect timing can introduce inter-symbol interference (ISI) and intercarrier-interference (ICI), which can degrade the system performance severely [5]. Several approaches $[6,7,8]$ have been proposed on the basis of using preamble symbols or using cyclic prefix and pilot subcarriers. For burst mode transmission such as wireless LAN, the method of using preamble symbols [6] is preferred for fast time synchronization. In this paper, we will discuss different synchronization schemes using the preamble specified in the IEEE 802.11 standard.

\section{System model}

The system model is based on the IEEE 802.11a standard [1], which employs the unscheduled packet-switched technique, the receiver needs to perform a start-of-packet detection (where the start of a packet is defined by the start of the preamble), frequency synchronization, and channel estimation before actually data bits can be decoded. To this end, the standard defines a preamble by which above tasks can be performed.

The first part of the preamble consists of 10 identical short training (ST) symbols with duration of $0.8 \mu$ s each. The ST sequence (STS) is used for coarse time and frequency synchronization. It is followed by two long training (LT) symbols, each of which is $3.2 \mu$ s plus a 1.6- $\mu$ s prefix called long guard interval (LGI) which precedes the long training symbol. The LT sequence (LTS) is used for fine time synchronization and channel estimation. The preamble is followed by the signal field and data. The total training length is $16 \mu \mathrm{s}$. A ST symbol consists of 12 subcarriers, which are modulated by the elements of the sequence $S$, given by [1]

$$
\begin{aligned}
& S_{-26,26}=\sqrt{13 / 6} \times\{0,0,1+j, 0,0,0,-1-j, 0,0,0, \\
& 1+j, 0,0,0,-1-j, 0,0,0,1+j, 0,0,0,0,0,0,0,-1-j, \\
& 0,0,0,-1-j, 0,0,0,1+j, 0,0,0,1+j, 0,0,0,1+j, 0,0\},
\end{aligned}
$$

where the multiplication by a factor of $\sqrt{13 / 6}$ is to normalize the average power of the resulting OFDM symbol, which utilizes 12 out of 52 subcarriers.

A long OFDM training symbol consists of 53 subcarriers (including a zero value at dc), which are modulated by the elements of the sequence $L$, given by [1]

$$
\begin{gathered}
L_{-26,26}=\{1,1,-1,-1,1,1,-1,1,-1,1,1,1,1,1,1,-1, \\
-1,1,1,-1,1,-1,1,1,1,1,0,1,-1,-1,1,1,-1,1,-1 \\
1,-1,-1,-1,-1,-1,1,1,-1,-1,1,-1,1,-1,1,1,1,1\} .
\end{gathered}
$$

In IEEE 802.11a, OFDM modulation method is used for data transmission. Consider an OFDM system employing $N$ subcarriers for the transmission of parallel data streams of width $N_{u}$. At the transmitter, the data stream is mapped into $N$ complex symbols in the frequency domain, including null data symbols for virtual subcarriers. These $N$ complex symbols are modulated on to the $N$ subcarriers by using Inverse Fast Fourier Transform (IFFT) to get a time domain complex OFDM symbol, which is represented as

$$
x(n)=\frac{1}{\sqrt{N}} \sum_{k=0}^{N-1} X(k) e^{\frac{j 2 \pi k n}{N}}, \quad n=0,1, \ldots, N-1
$$

where $X(k)$ denotes the data symbol in subcarrier $k$, $x(n)$ is the $n$th sample of the OFDM symbol. The last 
$N_{g}$ samples of the IFFT outputs are copied and added to form the cyclic prefix at the beginning of each OFDM symbol. In IEEE802.11a, $N$ is equal to $64, N_{u}$ is equal to 52 (including 4 pilot subcarriers) and $N_{g}$ is equal to 16 . The time domain symbols are interpolated, D/A converted, mixed with a carrier and transmitted.

At the receiver, the received signals are down-converted, filtered, A/D converted and decimated to reconstruct the baseband signals. The received signal transmitted through multi-path channels is expressed by

$$
r(n)=\sum_{i=0}^{L-1} x(n-\tau-i) h(i) e^{\frac{j 2 \pi f_{o} n}{N}}+w(n),
$$

where $h(i)$ is the complex channel impulse response (CIR), $L$ is the number of channel taps, $\tau$ is the time offset, $f_{o}$ is the carrier frequency offset and $w(n)$ is additive white Gaussian noise (AWGN). With the CIR confined to the cyclic prefix length, after removing the cyclic prefix the received signals are demodulated via FFT, the demodulated signal in subcarrier $k$ is given by

$$
Y(k)=X(k) H(k) e^{\frac{-j 2 \pi k \tau}{N}},
$$

where $H(k)$ is the channel frequency response in the $k$ th subcarrier, $e^{\frac{-j 2 \pi k \tau}{N}}$ is the phase rotation introduced by the time offset $\tau$. If the time offset is not in the range of cyclic prefix, it will cause ISI and ICI.

\section{Time synchronization algorithms}

Time synchronization in IEEE 802.11 is performed in two steps, i.e., coarse synchronization and fine synchronization. The task of coarse synchronization is to identify the preamble in order to detect a packet arrival. The term coarse timing is used here since it provides a reference point as to where to start the fine timing process.

Most timing synchronization schemes can be grouped into two categories: auto-correlation based algorithms and cross-correlation based algorithms. The former utilize the repetition structure of the training sequence or the guard intervals (GI) of OFDM symbols to acquire timing synchronization [6]. Cross-correlation based algorithms use the good auto-correlation property of training sequences to achieve a more precise timing $[9,10]$. There are some other synchronization methods, which use the maximum-likelihood (ML) mechanism to achieve better performance [11, 12]. However, their computational complexity usually is much higher than that of the above mentioned schemes, which makes them impractical. In what follows, we shall discuss different synchronization techniques (including cross-correlation, auto-correlation, energy detection schemes) that are feasible for practical implementations.

With the cross-correlation approach for the coarse time synchronization, we use one ST symbol as local reference signal at the receiver and cross-correlate it with the incoming signal

$$
M_{c s}(n)=\frac{\sum_{m=0}^{N_{c s}-1} r(n+m) S^{*}(m)}{\sum_{m=0}^{N_{c s}-1}|S(m)|^{2}},
$$

where $N_{c s}$ is the length of the correlation window, and (.)* stands for the conjugate operation.

For the fine time synchronization, we use the two LT symbols as local reference signal at the receiver and crosscorrelate them with the incoming signal

$$
M_{c l}(n)=\frac{\sum_{m=0}^{N_{c l}-1} r(n+m) L^{*}(m)}{\sum_{m=0}^{N_{c l}-1}|L(m)|^{2}} .
$$

where $N_{c l}$ is the length of the correlation window.

With the repeated short training symbols specified in IEEE802.11a, we can readily use the auto-correlation method to do initial coarse timing synchronization. We calculate the normalized auto-correlation timing metric between the received signal and itself with a delay of one short symbol $N_{s}$

$$
M_{\text {auto }}(n)=\frac{\sum_{m=0}^{N_{s}-1} r(n+m) r^{*}\left(n+m+N_{s}\right)}{\sum_{m=0}^{N_{s}-1}|r(n+m)|^{2}} .
$$

Next, we introduce an alternative coarse time synchronization scheme based on a measure of the energy of the incoming data. In this case, there is no need to have a specifically designed preamble or a training symbol. In the absence of data, the received signal only contains noise samples, which are uncorrelated to each other. The received signal can be expressed as

$$
r(n)=\left\{\begin{array}{ll}
w(n) & \text { in the absence of data } \\
x(n)+w(n) & \text { in the presence of data }
\end{array},\right.
$$

where $r(n), x(n)$ and $w(n)$ denote the received signal, transmitted data and noise, respectively. The accumulation of the energy of the signal over a window will result in small values for AWGN, and there will be a rise in the energy level after the start of the packet edge. This energy based detection method can be expressed as

$$
M_{e n}(n)=\sum_{m=0}^{N_{e n}-1}|r(n+m)|^{2},
$$

where $N_{e n}$ is the window length.

\section{Numerical results}

The performance of the discussed algorithms are evaluated by computer simulations. We use the following channel model adopted by the IEEE 802.11 working group [13]

$$
\begin{aligned}
h_{k} & =N\left(0,0.5 \sigma_{k}^{2}\right)+j N\left(0,0.5 \sigma_{k}^{2}\right) ; \\
\sigma_{k}^{2} & =\sigma_{0}^{2} \exp \left(-k T_{s} / T_{R M S}\right) ; \\
\sigma_{0}^{2} & =1-\exp \left(-T_{s} / T_{R M S}\right),
\end{aligned}
$$




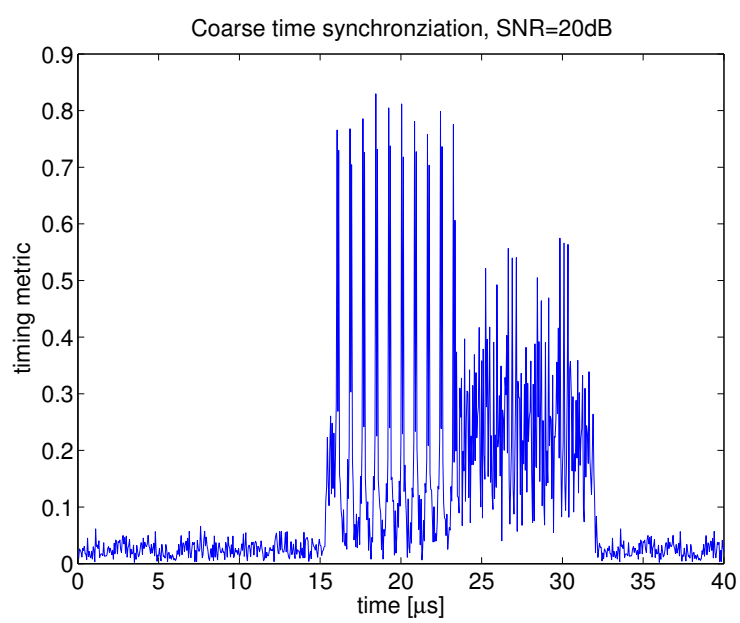

Figure 1: Coarse time synchronization with crosscorrelation using STS.

where $h_{k}$ is the complex channel gain of the $k$ th tap, $T_{R M S}$ is the RMS delay spread of the channel, $T_{s}$ is the sampling period, $\sigma_{0}^{2}$ is chosen so that the condition $\sum_{k} \sigma_{k}^{2}=1$ is satisfied to ensure same average received power. The number of samples to be taken in the impulse response should ensure sufficient decay of the impulse response tail, e.g., $k_{\max }=10 \times T_{R M S} / T_{s}$. The parameter setting in our simulations follows the IEEE 802.11a standard [1], i.e, the number of subcarriers is $N=64$, and $N_{u}=52$ subcarriers are used for transmission. One OFDM symbol duration is $3.2 \mu \mathrm{s}$, and sample duration is $3.2 / 64=0.05 \mu \mathrm{s}$. The RMS delay spread is set to be $T_{R M S}=50 \mathrm{~ns}$ and the sampling rate $f_{s}=1 / T_{s}=20 \mathrm{MHz}$. The short and long training symbols are generated according to (1) and (2), respectively. The SNR is set to $20 \mathrm{~dB}$ unless otherwise stated.

Fig. 1 illustrates the coarse time synchronization with the cross-correlation approach. The transmitter sends the preamble after $16 \mu$ s null transmission, i.e., the samples corresponding to the first $16 \mu \mathrm{s}$ only contain AWGN. $N_{c s}=16$ sample correlation is performed to calculate the metric expressed in (6). As can be seen from the figure, the cross-correlation between the received signal and the ST symbol as local reference signal produces 10 peak correlation values, with the first one at the starting point of the preamble, which is exactly at $16.05 \mu \mathrm{s}$. The peaks can be detected by using threshold or sorting out 10 maximum values at the output of the correlator.

Fig. 2 illustrates the coarse time synchronization with auto-correlation approach. The correlation window size in (8) equals the length of a ST symbol, i.e., $N_{s}=16$. It creates a plateau of the length of nine short symbols. The left cliff of the plateau corresponds to the starting point of ST symbol, and it can be detected by setting a threshold value. However, the spikes caused by the noise before the plateau can exceed the threshold value and leads to synchronization errors. This problem can be circumvented by processing the correlator output with some low pass

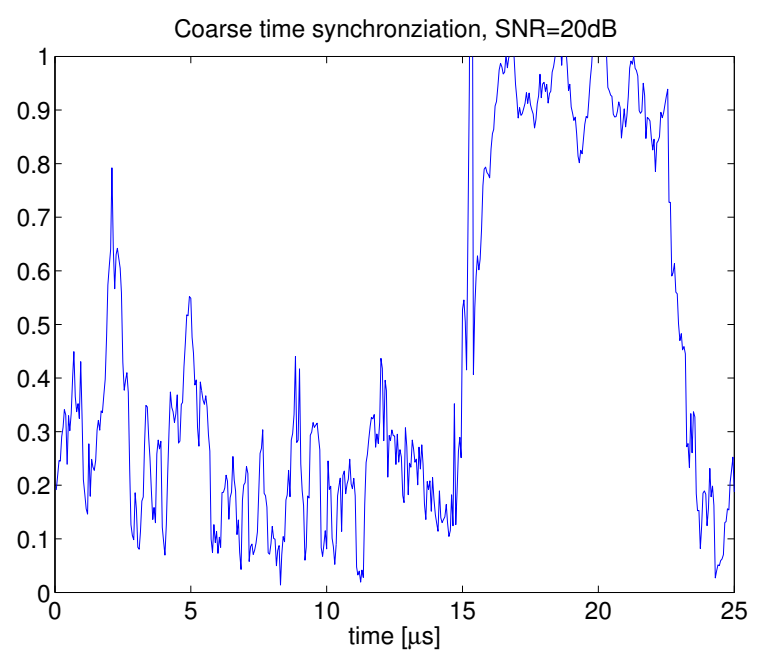

Figure 2: Coarse time synchronization with autocorrelation.

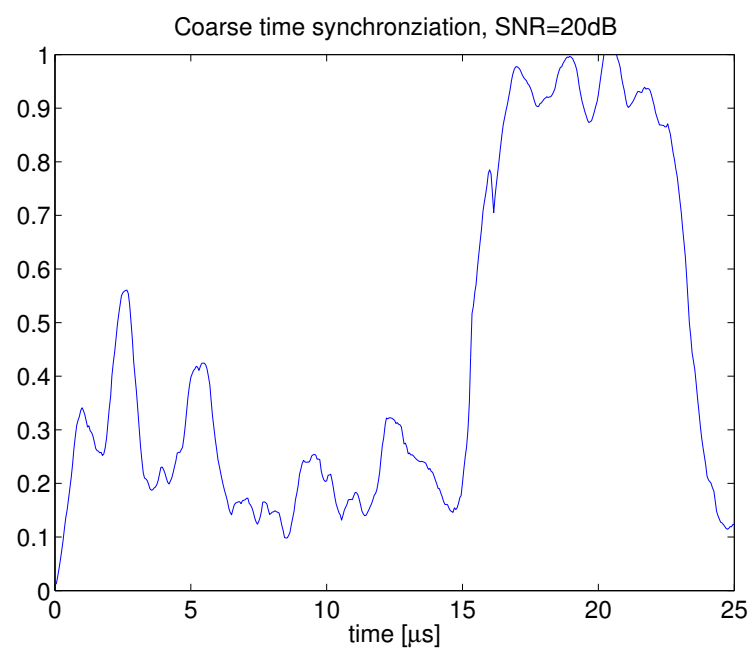

Figure 3: Improved time synchronization with low-pass filtering.

filter. Fig. 3 shows the results after applying a moving average low-pass filter to the original auto-correlation output. The spikes shown in Fig. 2 have been smoothed out, leading to more accurate time synchronization.

Coarse time synchronization based on the signal energy detection is demonstrated in Fig. 4. The window length in (10) is chosen to be $N_{e n}=16$. As shown in the figure, this energy detection approach is less prone to noise effect compared to the auto-correlation approach. All the investigated coarse time synchronization schemes have comparable computational complexity since they all involve a 16-sample correlation. However, we have observed that the cross-correlation approach yields the best synchronization performance, it is therefore a preferred solution.

Fig. 5 shows the fine time synchronization using the LTS. 


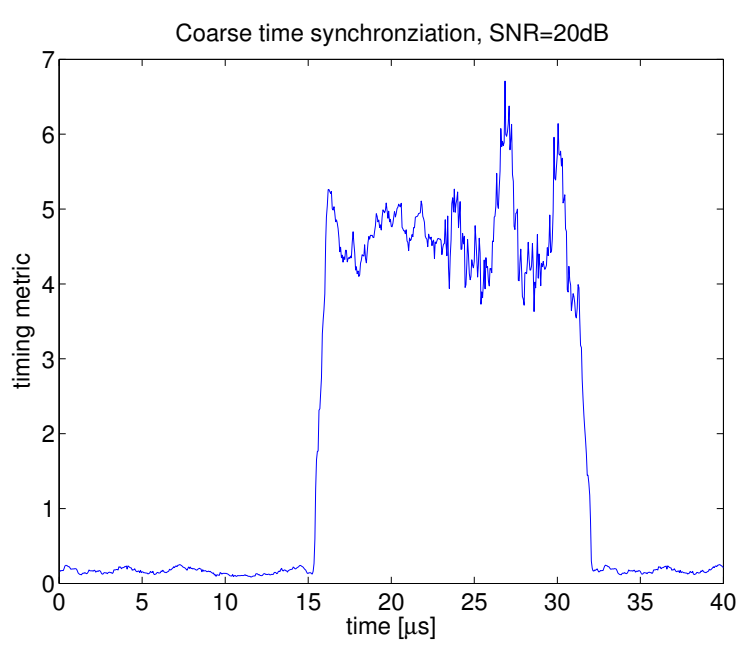

Figure 4: Coarse time synchronization based on the signal energy detection.

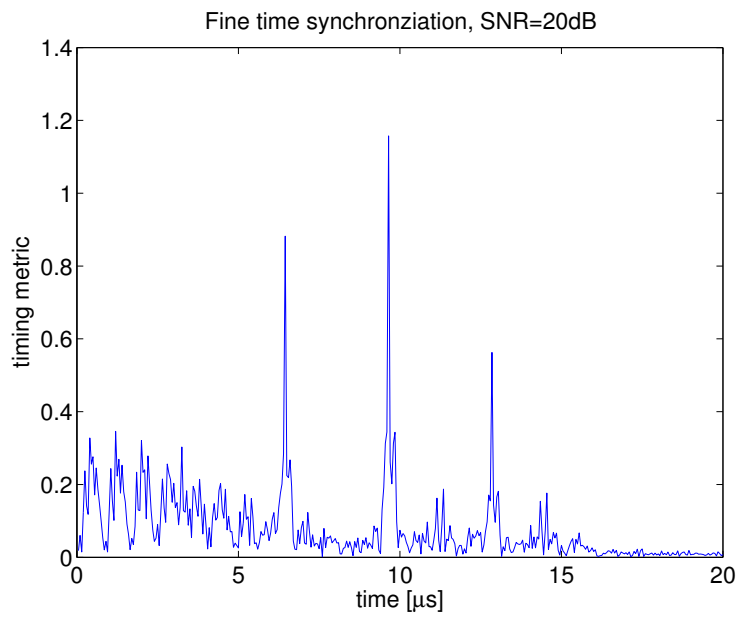

Figure 5: Fine time synchronization with cross-correlation using LTS.

In this case, the correlation window size in (7) equals the duration of two LT symbols, i.e., $N_{c l}=128$. It gives one peak correlation value, indicating the starting point of the LTS, which occurs at $9.6 \mu$ s after the start of the preamble.

\section{Conclusions}

In the WLAN standard adopted by the IEEE 802.11 standard group, each data packet starts with a preamble, which consists of 10 short training symbols followed by 2 long training symbols. We have discussed different timing synchronization algorithms using the short and long training symbols in this paper. Among different equal-complexity schemes, such as the ones using auto-correlation, crosscorrelation, energy detection, we observed that the crosscorrelation approach gives the most reliable performance. The work presented in this paper provides a useful reference for the practical implementation of the IEEE 802.11 products.

\section{Acknowledgement}

This work was supported by the Strengthen All Island (SAI)

- Mobile Wireless Future project.

\section{References}

[1] IEEE Std 802.11a-1999, Part 11: Wireless LAN Medium Access Control (MAC) and Physical Layer (PHY) Specifications - High-speed Physical Layer in the $5 \mathrm{GHz}$ Band, IEEE, 1999.

[2] L. Wei and C. Schlegel. "Synchronization requirements for multi-user OFDM on satellite mobile and two-path Rayleigh fading channels". IEEE Trans. Comm., vol. 43, no. 234, pp. 887-895, Feb/Mar/Apr 1995.

[3] T. Pollet and M. Moeneclaey. "Synchronizability of OFDM signals", In Proc. IEEE GLOBECOM'95, vol. 3, pp. 2054 2058, Nov. 1995.

[4] W. D. Warner and C. Leung. "OFDM/FM frame synchronization for mobile radio data communication". IEEE Trans. Vehicular Tech., vol 42, no. 3, pp. 302-313, August 1993.

[5] M. Speth, S. A. Fechtel, G. Fock and H. Meyr. "Optimal receiver design for wireless broad-band systems using OFDM - Part 1", IEEE Trans. on Communi., vol. 47, no. 11, Nov. 1999.

[6] T. M. Schmidl and D. C. Cox. "Robust frequency and timing synchronization for OFDM", IEEE Trans. on Commun., vol. 45, no. 12, pp. 1613-1621, Dec. 1997.

[7] J.-J. v. d. Beek, M. Sandell, and P. O. Brjesson. "ML estimation of time and frequency offsets in OFDM systems", IEEE Trans. Signal Processing, vol. 45, pp. 1800-1805, 1997.

[8] Minn, M. Zeng, and V. K. Bhargava. "On timing offset estimation for OFDM systems". IEEE Commun. Letters, vol. 4, pp. 242-244, 2000.

[9] K-W Yip; Y-C Wu; T-S Ng. "Timing synchronization analysis for IEEE 802.11a wireless LANs in frequency nonselective Racian fading environments", IEEE Trans. on Wireless Communications, vol 3, no. 2, pp. 387-394, Mar., 2004.

[10] A. N. Mody and G. L. Stuber. "Synchronization for MIMO OFDM systems". Proc. IEEE Global Commun. Conf., vol. 1, pp. 509-513, Nov. 2001.

[11] Y.-C. Wu, K-W. Y, T-S. Ng, E. Serpedin. "Maximumlikelihood symbol synchronization for IEEE 802.11a WLANs in unknown frequency-selective fading channels". IEEE Trans. Wireless Communications, vol. 4, no. 6, pp. 2751-2763, Nov. 2005.

[12] E.G. Larsson, G. Liu, J. Li, and G.B. Giannakis. "Joint symbol timing and channel estimation for OFDM based WLANs". IEEE Commun. Letters, vol. 5, no. 8, pp. 325327, Aug. 2001.

[13] B. O'Hara, A. Petrick. IEEE 802.11 Handbook: A Designer's Companion. 2nd Edition, IEEE, 2005. 